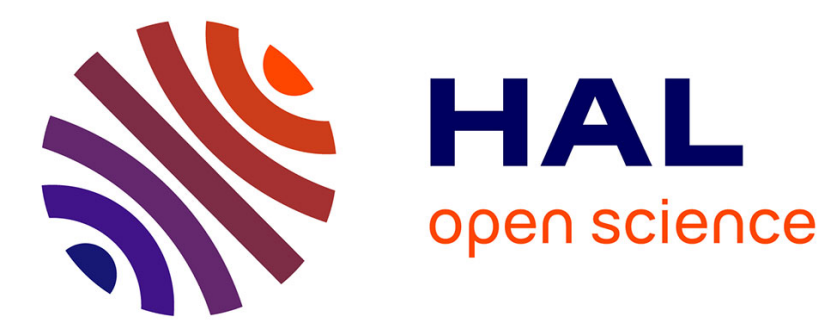

\title{
Displacement Sensor Based on Radar Cross-Polarization Measurements
}

\author{
Etienne Perret
}

\section{To cite this version:}

Etienne Perret. Displacement Sensor Based on Radar Cross-Polarization Measurements. IEEE Transactions on Microwave Theory and Techniques, 2017, 65 (3), pp.955-966. 10.1109/TMTT.2016.2638842 . hal-02065457

\section{HAL Id: hal-02065457 https://hal.science/hal-02065457}

Submitted on 1 Jul 2020

HAL is a multi-disciplinary open access archive for the deposit and dissemination of scientific research documents, whether they are published or not. The documents may come from teaching and research institutions in France or abroad, or from public or private research centers.
L'archive ouverte pluridisciplinaire HAL, est destinée au dépôt et à la diffusion de documents scientifiques de niveau recherche, publiés ou non, émanant des établissements d'enseignement et de recherche français ou étrangers, des laboratoires publics ou privés. 


\title{
Displacement Sensor-based on Radar Cross- Polarization Measurements
}

\author{
Etienne Perret, Senior Member, IEEE
}

\begin{abstract}
This article presents the feasibility of measuring submillimeter displacements of objects in real environments using simple resonant radar targets. These scatterers, also known as chipless tags, have their own radar signature that can be used not only for identification but also for sensing. The chipless RFID is a highly promising technology since it paves the way for the development of easily implementable communication systems where tags have costs similar to barcodes. Object displacements are extracted by just evaluating at different times the variation of the signals backscattered in cross polarization from the tags. Based on this analog RFID sensing principle, an analytical model is developed to perform the direct derivation of the displacements. Therefore, the approach does not require complex and specific calibration procedures based on lookup table for compensating variations due to the presence of surrounding unknown objects. It is possible to add this functionality to already designed chipless RFID tag operating in cross-polarization without decreasing their coding capacity. Experimentations inside and outside anechoic chamber have been done, and very good agreement was obtained with simulations. It has been proved that sub-millimeter displacements can be monitored with this approach. Chipless tag displacement of $0.5 \mathrm{~mm}$ with less than $\pm 45 \mu \mathrm{m}$ error can be detected in real environment for a read range of up to $40 \mathrm{~cm}$.
\end{abstract}

Index Terms - Chipless RFID, displacement measurement, radar cross section (RCS), RFID, sensor.

\section{INTRODUCTION}

$\mathrm{I}^{\mathrm{s}}$ DENTIFICATION and physical quantity capture are crucial issues in many areas of the society. High-speed metrology, position sensing, and fluid level sensors are typical engineering applications where accurate and fast displacement measurement is required. This is also true in the field of items tracking, as well as for medical applications. The Radio Frequency Identification (RFID) is even more utilized because its tags do not need to be powered by a battery. This facilitates its development by preventing and responding to challengingly constraints such as the question of prize, life time, integration, etc. [1]. However, a technology that can combine the flexibility of RF waves utilization and the extremely low cost of barcodes could have a bright future. It is why Chipless RFID could be the solution even for sensor applications [2].

Traditional UHF (Ultra-high Frequency) RFID technology, without adding any specific analog or digital components, has been widely used for various applications in instrumentation [3]-[5] such as displacement measurement [6], [7]. Significant results have been obtained, but for such applications, performances are directly limited by the problem of tag's

Etienne Perret is with the University of Grenoble Alpes - LCIS, 50 rue Barthélémy de Laffemas - BP 54, 26902, Valence Cedex 9 - France. He is also with the Institut Universitaire de France. (etienne.perret@lcis.grenobleinp.fr). reproducibility, more especially due to chip sensitivity variations. The approaches are based on power activation or delta radar cross section (RCS) measurements that are both necessary quantities linked to the chip characteristics. If these characteristics are not perfectly reproducible from one chip to the other, additional errors will directly impact the accuracy of the physical quantity to measure [8]. This will considerably limit the sensor application [9]. For example, the nonlinear behavior of the chip makes the extraction part of the physical quantity to be monitored more complicated [10]. That is all the more true when only the chip characteristic knowledge is incomplete, or can be obtained with poor accuracy. These uncertainties (such as the variation of the input impedance with the power, which is not given by the chip manufacturers [11]) are still not well predictable in terms of effects on the attainable accuracy for analog sensing purpose. Basically, RFID chips are not designed to monitor a physical quantity. Moreover, it is well known that the radar approach used to interrogate UHF RFID tags, and so to monitor the physical quantity, exhibits large uncertainties with a strong sensitivity on the reader [12], [13]. Further studies are needed to assess the true performance of RFID systems (the tags but also the reader part) in term of accuracy for analog sensing [8]. It is why a solution where we can get grid of the chip will provide better performance in terms of sensitivity [2], [9]. Thus, just like for conventional RFID, the question of adding the sensor application in chipless arises [14]-[18]. The measuring principle, also based on a radar approach, remains the same and it can easily be transposed to chipless tags. Thus, without adding specific electronic components, it is possible to effectively use chipless RFID techniques. For instance, in literature, objects deformation measurement based on the concept of UHF RFID grid has been done in [7]. But, the obtained accuracy of the order of ten centimeters could be too low for some applications. It can be seen that for more specific configurations, a sub-millimeter resolution can be obtained with a chipless approach. This is possible only if the tag used to realize the measurement can be also associated to an identifier. It is therefore possible, like in UHF RFID, to set up a wireless sensor network without chip to make low cost and potentially dispose sensor tags [19]. In addition to the accuracy improvement, taking off the chip allows reducing the cost, increasing the duration of life, and the mechanical robustness in terms of vibration and temperature [2].

Optical interferometers give very good performance with resolution ranging from micrometer to sub-nanometer [20], [21]. However, a direct line of sight is needed. It is also difficult to use this approach if measurements on different 
locations, or on different objects are required to be done at the same time. This is particularly true when the object's positions are changing from time to time. It is the reason why RF approaches based on radar equipment are also used for these applications. Less performance is obtained even if submillimeter resolution can be reached [22], [23]. Like with an optical solution, measurements of displacement of water level and metal plate location can be done but it is also possible to make displacement measurements through objects such as clothes. This explains why different UWB radars have been developed for medical applications, i.e. for non-contact measurements to monitor respiration and tidal volume in human subjects [24], [25]. Unlike RFID solutions, it is not necessary to place a tag on the object to monitor it. This presents a great advantage; however, without any tag or label, it is not possible to identify the object at the same time as to do the deformation measurement. It is clearly a drawback in many applications, especially when multiple characterizations are required at the same time. Indeed, in the case of UWB radar approaches, measurements cannot be done locally like with optical solution, and this low directivity can dramatically cause some errors of detection when multiple objects, placed in front of the radar antenna, move. In such configuration, RFID approach can resolve this problem since tags can now be attached to the object to be monitored. In this article, for the first time, displacement measurements based on a chipless RFID solution is introduced. Through the utilization of a potentially low cost chipless tag [2], [26], displacement measurements that could be done in a non-fixed environment, as schematized in Fig. 1, is presented. This approach is compatible with multiple reading all at the same time. In comparison with [7], lower distances are attainable, but much higher resolution can be obtained with a very basic, potentially disposable tag, i.e. without any chip. Moreover, the tag ID and the information on the tag displacement can be monitored exactly at the same time. Note that this aspect cannot be done in UHF RFID due to the use of an anti-collision protocol which results in the interrogation of each tag one after the other.

The technique presented here is based on a classical radar approach coupled with the use of chipless RFID tags. The presence of the tag attached to the object allows 1) to measure the displacements of a precise, localized part of the object (such as with an optical approach) even in the presence of other movable and opaque objects; and 2) to identify the hosting object. Because of these two functionalities, this approach can be used for deformation survey. In that case, a configuration with multiple tags can be used to monitor the displacement of different parts of the surface under concern. We will see that this sensing functionality can be implemented on already designed chipless RFID tag operating in crosspolarization. Moreover, this option does not reduce the coding capacity of these tags. Indeed, unlike other approaches where some resonators must be reserved for sensing activity [14][18], in the proposed method, each resonator is used for identification as well as for displacement sensing. Contrary to optical solutions, the measurement can be done through opaque dielectrics that can be placed between the reader and the hosting object. It means that non-line-of-sight access is needed. The measurements of all the different tags can be done simultaneously, with only one reader classily used for chipless RFID applications [27].

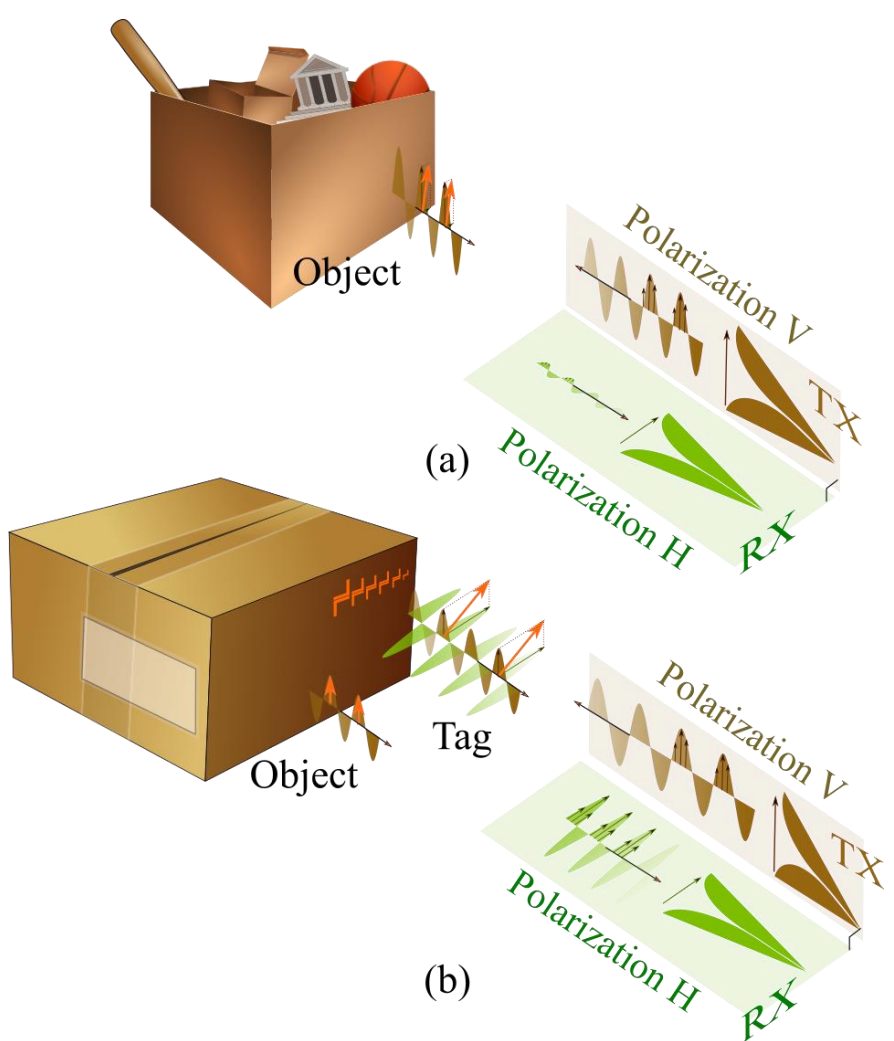

Fig. 1. Principle of the chipless RFID system used to monitor deformations. The TX antenna sends a signal in vertical polarization while the RX antenna operates in horizontal polarization. (a) The backscattered wave from an object keeps the same orientation than the incident wave (vertical polarisation). (b) A depolarizing tag creates a backscattered wave with a significant contribution perpendicular to the incident wave.

In this article, the possibility of using chipless tags to measure the displacement of tagged objects, with a special focus on the maximum resolution that can be obtained, is presented. The approach is based on cross polarization measurement, which makes it possible to isolate the hosting object displacement form the other objects that can be potential movable.

The paper is organized as follows: Section II presents the theory and the expressions derived to monitor tags displacements in various scenarios; Section III studies the validity of the introduced model based on simulations; Section IV presents the experimental results with depolarizing tags inside and outside anechoic chamber; and finally, Section V draws the conclusions.

\section{II.CONCEPT OF CROSS-POLARIZATION MEASUREMENT FOR CHIPLESS DISPLACEMENT SENSOR}

The concept of depolarized tag based on RF Encoding Particles (REP) has been introduced in [28]. As described in Fig. 1, simple scatterers have been designed to backscatter the electromagnetic (EM) field in an orthogonal direction compared to the incident orientation [see Fig. 1(b)]. It has been proven that ID recovery can be done without any calibration process which means without any information on the hosting object (material, shape, distance, etc.) [28], [29]. This significant improvement in terms of reading flexibility comes from the fact that usual objects do not depolarize EM waves [see Fig. 1(a)]. If the reader does the acquisition of the backscatter field in a direction perpendicular to the incident 
field, the signal to noise ratio is significantly increased. This basic principle can be favorably used to do displacement measurements in a movable environment as the one depicted in Fig. 1.

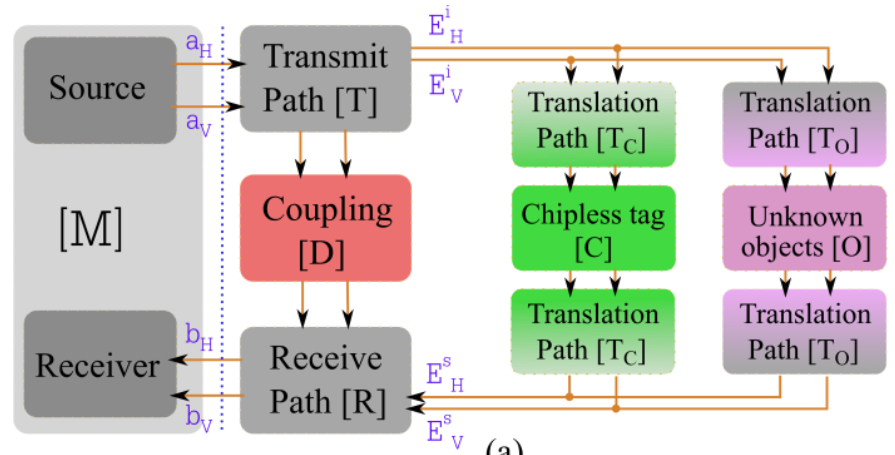

(a)

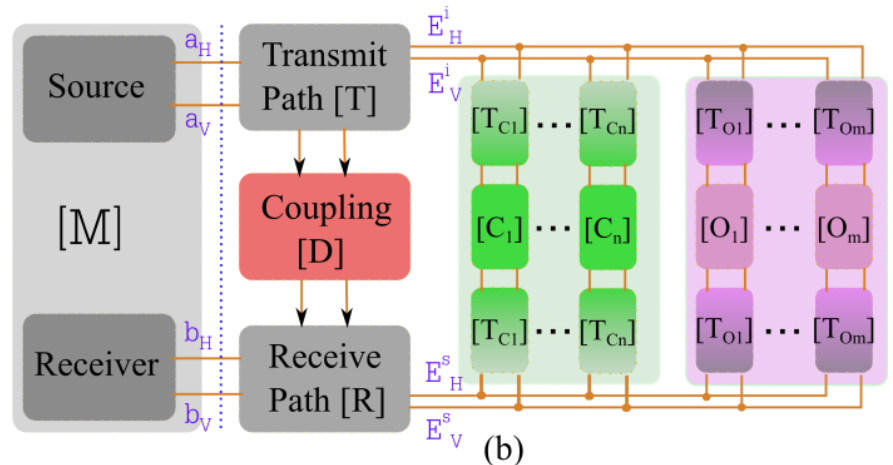

Fig. 2. Channel model of the system, accounting for displacement measurements. (a) Basic configuration with one chipless tag and one object. (b) Generalisation to $n$ different tags and $m$ unknown objects.

\section{A. Analytical model of tag and object displacements - cross- polarization configuration}

For the sake of clarity, same notations in [28] are used. Symbols $a_{h, v}$ and $b_{h, v}$ denote the transmitted and received power waves in horizontal $(h)$ and vertical $(v)$ polarizations, respectively. Symbols $E^{i, s}$ denote the incoming and backscattered field at the tag.

A block diagram of the detection system has been introduced in [28, Fig. ${ }^{\circ}$ 5]. The detection system is represented by the block denoted by [M]. The block corresponding to the tag (respectively the object) is [C] (respectively [O]). Note that the channel model in [28, Fig. ${ }^{\circ}$ ] corresponds to a simplified version of the one presented Fig. ${ }^{\circ}$. Indeed, the translation paths $\left[\mathrm{T}_{\mathrm{C}}\right]$ and $\left[\mathrm{T}_{\mathrm{O}}\right]$ which represent displacement variations to be monitored, are not taken into account in [28, Fig. 5].

$$
\left[T_{C}\right]=\left[\begin{array}{cc}
e^{-j k d_{C}} & 0 \\
0 & e^{-j k d_{C}}
\end{array}\right], \quad\left[T_{O}\right]=\left[\begin{array}{cc}
e^{-j k d_{O}} & 0 \\
0 & e^{-j k d_{O}}
\end{array}\right]
$$

$k$ is the phase constant, $d_{C}$ is the tag's displacement, and $d_{O}$ is the object's displacement (see Fig. 3). $D$ is the distance between reader's antennas and the tag. Following the same derivation than in [28] gives:

$$
M_{v h}^{\prime\left(d_{C}, d_{O}\right)}=I_{v h}+R_{v v}\left(e^{-j 2 k d_{C}} C_{v h}+e^{-j 2 k d_{O}} O_{v h}\right) T_{h h} .
$$

$I_{v h}$ is the direct coupling between antennas, also called isolation measurement $\left(M_{v h}=I_{v h}\right.$ if neither tag [C], nor objects [O] are in the front of the antennas). With the assumptions that 1) the surrounding objects in the detection area do not de-polarize incident waves; and 2) there is a presence of depolarizing tags near their resonance frequencies, then $\left|C_{v h}\right| \gg\left|O_{v h}\right|$. In this case, one can rewrite (1), into (2) as follows:

$$
M_{v h}^{\prime\left(d_{C}\right)}=I_{v h}+e^{-j 2 k d_{C}} R_{v v} C_{v h} T_{h h}
$$
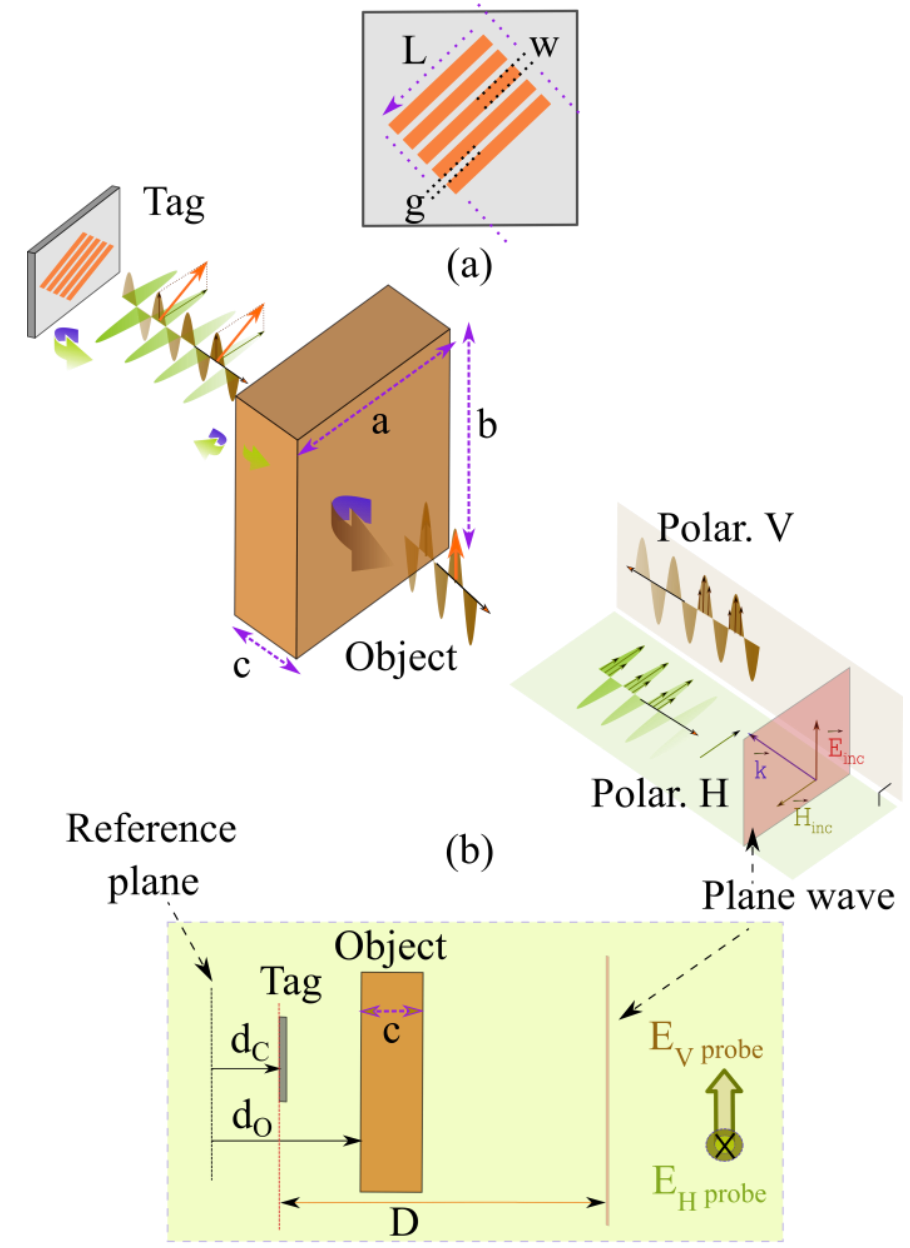

(c)

Fig. 3. Simulation set up. (a) Depolarizing tag (single resonator) based on shorted dipoles oriented at $45^{\circ}$, realized on Rogers RO4003, $L=23 \mathrm{~mm}, g=$ $0.5 \mathrm{~mm}, w=2 \mathrm{~mm}$. (b) Configuration with one tag and one object (dielectric plate of Rogers RO4003, $\left.\varepsilon_{r}=3.55, \tan \delta=0.0027\right), a=b=150 \mathrm{~mm}, c=10 \mathrm{~mm}$. (c) Side view of a tag behind the plate of $10 \mathrm{~mm}$ thickness, $d_{0^{-}} d_{C}=50 \mathrm{~mm}$.

Indeed, at the resonance frequency, depolarized tags present a maximum on their component $\left|C_{v h}\right|$. Outside the resonances, (1) has to be considered. All these assumptions will be proven in the simulation and measurement part.

Note that when $d_{C}=O$, which corresponds to $M_{v h}^{\prime(0)}$, [28, eq. (9)]is directly obtained form (2). Finally, one can obtain (3) using (2) in order to extract the cross-polarization scattering term of a chipless tag with three measurement results, which are $I_{v h}, M^{\prime}(0)$, and $M_{v h}^{\prime\left(d_{C}\right)}$.

$$
d_{C}=\frac{1}{2 k} \ln \left(\frac{M_{v h}^{\prime(0)}-I_{v h}}{M_{v h}^{\prime\left(d_{C}\right)}-I_{v h}}\right) \text {. }
$$

Note that (3) is valid only when $\left|C_{v h}\right| \gg\left|O_{v h}\right|$, which means only around the tag's resonance frequencies, that is to say the frequencies where $\left|M^{\prime}{ }_{v h}^{(0)}\right|$ is maximum (peak apex). Under these hypotheses, one significant result is that (3) can be used in a more general case, that is to say when the tag is surrounding by multiple unknown objects that can move [see Fig. 2(b)]. More exactly, in practice, even after that the isolation measurement $I_{v h}$ has been done, it is possible to 
move or to add new objects in the reading zone; (3) can still be used to retrieve the displacement $d_{C}$ of the tag.

Now, it is time to consider different practical cases. If $\left|O_{v h}\right| \gg\left|C_{v h}\right|$, which can correspond to the absence of the depolarizing tag, or when the interrogation frequency is not in the range of the tag resonance frequencies, a similar expression as (3) can be derived. Indeed, $d_{O}$ can be extracted based on the measurements of $I_{v h}, M_{v h}^{\prime(0)}$, and $M_{v h}^{\prime\left(d_{O}\right)}$. Note also that in the latter case, the validity of the expression is different from the one corresponding to (3), there is no limitation on the frequency range, but this expression cannot be generalized when multiple objects are in the front of the reader. Contrary to (3), it is definitely not interesting for this paper's application.

The case when the tag is attached to the object, that is to say when $d_{C}=d_{O}+c$, can also be considered. In that particular case, an expression similar to (3) can be used without any restriction on the frequency range even outside the tag resonance frequencies. But unfortunately, if another object is added to the reading zone, the expression is no longer applicable. Only (3) with its specific limitations (extraction of $d_{C}$ at the resonance frequencies) can be used in such more general configuration.

For focused application, the most important relation is (3) with its associated hypothesis. Indeed, as depicted Fig. 1, (3) made it possible to consider displacement measurements of a tag attached to an object surrounding by multiple objects that can arbitrary move during the measurement. In practice, to implement (3), the calibration process is very simple: only the direct coupling from the reader's transmitting to receiving antenna in cross-polarization $I_{v h}$ has first to be measured. Then it is possible to retrieve $d_{C}$ just by evaluating the signal $M_{v h}^{\prime\left(d_{C}\right)}$ backscattered from the tag at different times. Note that using (3), the extraction of the sensing data is straightforward, and does not require any calibration curves (like in most cases [30]-[34]) that give the relation between $M_{v h}^{\prime\left(d_{C}\right)}$ and the physical quantity. Note that possible interactions between the tag and the object due to their relative positions (for instant coupling effects, or the appearance of multiple reflections) are not considered in the analytical model illustrated Fig. 2. Indeed, here, the object $O$ and tag coefficient $C$ are supposed to be independent. Most of these interactions are difficult to consider with an analytical approach like the one used in this paper. However, one particular interaction that often occurs in practice will be considered, the case when the object is placed between the tag and the reader antenna [see Figs. 3(b) and (c)]. The difficulty is that such configuration is the presence of multiple reflections, especially between the object and the tag, but also the direct reflection due to the object that will attenuate the useful signal coming from the tag.

\section{B. Analytical model of tag and object displacements - co-} polarization configuration

To underline the interest of using a measurement method based on depolarizing tags, the expression of the backscattered signal in co-polarization $\left(M_{v v}^{\prime}\right)$ will be derived. Again, it is considered that the cross-polarization terms $R_{v h}$ and $T_{v h}$ are relatively low compared to the co-polarization terms $R_{v v}$, and $T_{h h}$. Similarly, in co-polarization, the term $M_{v v}^{\prime\left(d_{C}, d_{O}\right)}$ may be expressed:

$$
M_{v v}^{\prime\left(d_{C}, d_{O}\right)}=I_{v v}+R_{v v}\left(e^{-j 2 k d_{C}} C_{v v}+e^{-j 2 k d_{O}} O_{v v}\right) T_{v v}
$$

Let us note $M_{v v}^{\prime\left(d_{C}=0, d_{O}=0\right)}=M_{v v}^{\prime(0,0)}=M_{v v}^{\prime}$. The indices $v v$ relate to the co-polarization configuration. From (4), and by considering the two different cases $M^{\prime}{ }_{v v}$ and $M_{v v}^{\prime\left(d_{C}, d_{O}\right)}$, (5) can be derived :

$$
\frac{M_{v v}^{\prime\left(d_{C}, d_{O}\right)}-I_{v v}}{M_{v v}^{\prime}-I_{v v}}=\frac{e^{-j 2 k d_{C}} C_{v v}+e^{-j 2 k d_{O}} O_{v v}}{C_{v v}+O_{v v}} .
$$

Similarly to the cross-polarization configuration, several cases can be considered:

1 - The tag is placed on the object, that is to say $D=0$, and $d_{C}=d_{O}=d$, one can rewrite (5) into (6) as follows:

$$
d=\frac{1}{2 k} \ln \left(\frac{M_{v v}^{\prime}-I_{v v}}{M_{v v}^{\prime(d)}-I_{v v}}\right)
$$

Similarly, (6) can be applied without any restriction on the frequency range but only then at least one object [O] is facing the reader. However, the object hides the tag signature, making the tag ID recovery more difficult [28]. Last remark, (6) is not linked to the presence of the tag. For example, we would obtain (6) even if $C_{v v}=0$.

2- $d_{C} \neq d_{O}$, the tag and the object are considered to be independent. This also corresponds to the case when an object has been added after that the isolation measurement $I_{v h}$ has been done. In that configuration, it is not possible to extract $d_{C}$ form (5) like previously. If $\left|C_{v v}\right| \ll\left|O_{v v}\right|$ will be considered (6) can be used to determine the object displacements $d_{O}$ (with $\left.d=d_{o}\right)$.

In conclusion, in co-polarization it is not possible to extract $d_{C}$ in an environment containing multiple potentially movable objects. It is obvious from these derivations that displacement measurements can be realized due to the use of depolarizing tags and with a detection system sensitive to the crosspolarization.

\section{Generalization of the displacement sensor approach}

The objective here is to show that the presented approach is also compatible with multi-tag reading. Indeed, it is possible in chipless RFID to interrogate several tags at the same time. In such case, a specific encoding technique that allows to address the problem of collision in chipless has to be used [19]. To separate the response of one tag from the other, a principle of discrimination in frequency can be implemented.

To obtain the expression that takes into account the presence of multiple tags, the terms $C_{v h(1)}, C_{v h(2)}, \ldots, C_{v h(n)}$ relative to the presence of each of the $n$ tags [see Fig. 2(b)] have to be added in (2). It is noted that $d_{C(i)}$ the distance between the tag number $i$ and the reader. Equation (7) can be derived from (2) and from the channel model presented Fig. 2(b).

$$
\begin{gathered}
M^{\prime}{ }_{v h}^{\left(d_{\left.C(1) \cdots, d_{C(n)}\right)}\right.}(f)=I_{v h}+R_{v v}\left(e^{-j 2 k d_{C(1)} C_{v h(1)}+\cdots+}\right. \\
\left.e^{-j 2 k d_{C(n)}} C_{v h(n)}\right) T_{h h}, \forall f=\left\{f_{i}\right\}, i=[1, \ldots, n]
\end{gathered}
$$

To simplify (7), it is considered that each tag $C_{v h(i)}$ has been designed to have one specific frequency of resonance $f_{i}$. Each of them is supposed to be frequently separated of at least $100 \mathrm{MHz}$ to avoid interferences between two closed resonances. Under these hypotheses, at the frequency $f_{i}$, we have $\left|C_{v h(i)}\left(f_{i}\right)\right| \gg\left|C_{v h(1)}\left(f_{i}\right)\right|, \ldots,\left|C_{v h(n)}\left(f_{i}\right)\right|$. Indeed, as frequency domain chipless tags are designed to behave like 
high-Q resonators, there is typically a difference of at least $15 \mathrm{~dB}$ in the backscattered E-field between the maximum of the signal at the tag resonance frequency (the peak apex which corresponds to the contribution of the chipless tag antenna mode) and the level of the signal outside the resonance frequency range (the structural mode contribution). Fig. 4 illustrates this characteristic that is used to derive (8).

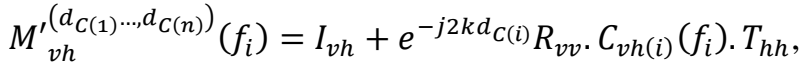

Equation (8) derived from (7) allows extracting the crosspolarization term of the chipless tag response $M^{\prime}{ }_{v h}^{\left(d_{C(1) \ldots,} d_{C(n)}\right)}\left(f_{i}\right)$ at the frequency of resonance $f_{i}$. Note that in (8), each term is frequency dependent, but for sake of clarity, this dependency noted $\left(f_{i}\right)$ is specified only for the most significant terms.

Equation (9), which corresponds to the tag displacement $d_{C(i)}$, can be derived from (8). Note that thinks to anticollision encoding scheme, the extraction of the displacement $d_{C(i)}$ for each tag $i$ in the reading zone can be obtained. The expression is similar to (3).

$$
d_{C(i)}=\frac{1}{2 k} \ln \left(\frac{{M_{v h}^{\prime(0)}}^{(0)}\left(f_{i}\right)-I_{v h}}{{M^{\prime}}_{v h}^{\left(d_{\left.C(1) \cdots, d_{C(n)}\right)}\left(f_{i}\right)-I_{v h}\right.}}\right)
$$

One interesting remark concerning (9) is that the terms $M^{\prime}{ }_{v h}^{\left(d_{C(1) \cdots,} d_{C(n)}\right)}(f),{M^{\prime}}_{v h}^{(0)}(f)$ and $I_{v h}$ contain the information of very tag displacements $d_{C(i)}$ placed in front of the reader. One can access to these values just by plotting $d_{C(i)}$ versus the frequency. Note also that these values $d_{C(i)}$ can be obtained all at the same time (for instance, if a short pulse is used to interrogate the tag), which is clearly different from analog sensing approaches based on UHF tags where the communication protocol imposes to interrogate one tag after the other.

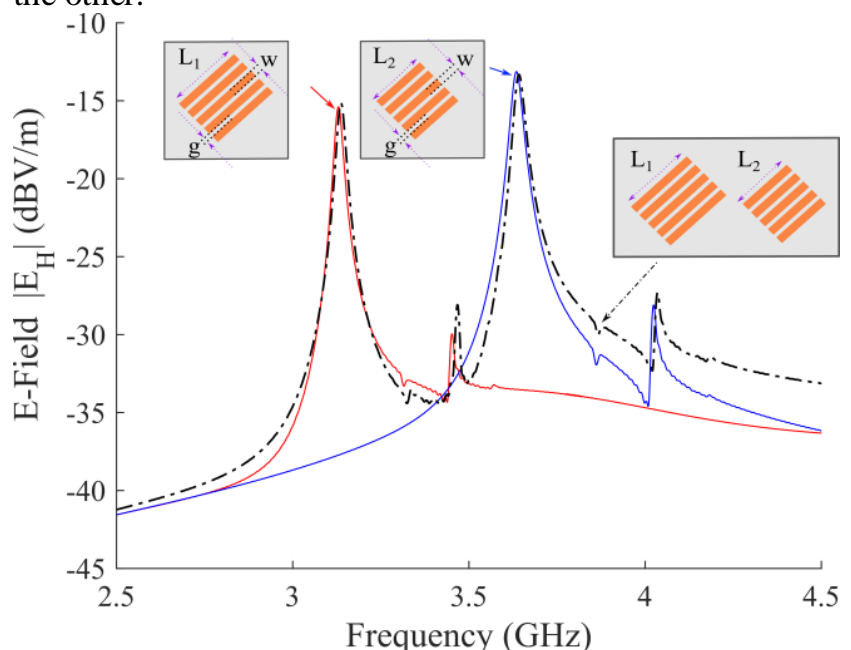

Fig. 4. Depolarizing tag based on shorted dipoles oriented at $45^{\circ}$. Simulation of the E-Field response in cross-polarization in three configurations: a resonator with $L_{l}=21.17 \mathrm{~mm}$, a resonator with $L_{2}=24.78 \mathrm{~mm}$, and both resonators together. The others tag dimensions are $w=2 \mathrm{~mm}$ and $g=0.5 \mathrm{~mm}$.

\section{Simulation Results}

Simulation has been done using CST Microwave Studio for the set up depicts in Fig. 3. Depolarizing tag based on shorted dipoles oriented at $45^{\circ}$ [see Fig. 3(a)] have been used for the study. The object under consideration is a reflective dielectric plate of $10 \mathrm{~mm}$ thickness, and 3.55 of permittivity [see Fig. 3(b)]. A plane-wave simulation of the structure has been done. E-field $E_{\text {inc }}$ is applied in the vertical direction of the proposed structure. To record the backscattered EM field (magnitude and phase) in the vertical direction $E_{V}$ and the horizontal direction $E_{H}$, CST "far-field probes" were used as seen in Fig. 3(c). Note that in such simulation, the direct coupling $I_{v h}$ (or $\left.I_{v v}\right)$ is null. Also, in such configuration, $M_{v h}^{\prime\left(d_{C}\right)}(f)=$ $E_{H}\left(f, d_{C}\right)$, and $M_{v v}^{\prime\left(d_{C}\right)}(f)=E_{V}\left(f, d_{C}\right)$. Expressions (6) (crosspolarization configuration) and (9) (co-polarization configuration) can be directly derived for various physical values of $d_{C}$ and $d_{o}$.

Simulations will be used to check the validity of the expressions introduced in Section II. Tag displacements are shown in Fig. 5. The E-field response of the tag is plotted Fig. 4. In that study, six displacements from $0.5 \mathrm{~mm}$ to $3 \mathrm{~mm}$ (with a step of $0.5 \mathrm{~mm}$ ) has been simulated. By using (3) and (6), a perfect agreement is observed between the expected values and the simulation results for the entire frequency band. In this simple configuration, the direct coupling is null and (2) and (5) are strictly true regardless the frequency of operation.

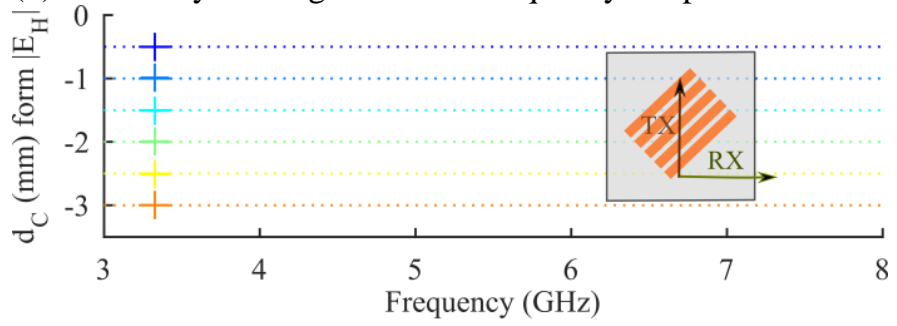

(a)

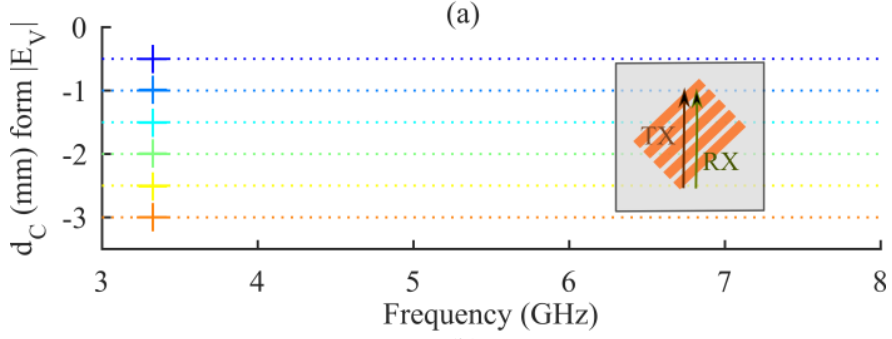

(b)

Fig. 5. Displacements of a depolarizing tag (single scatterer) based on shorted dipoles oriented at $45^{\circ}$ operating at $3.34 \mathrm{GHz}$. (a) $d_{C}$ is derived from the crosspolarized E-field $E_{H}$ tag response using (3). (b) $d_{C}$ is derived from the copolarized E-field $E_{V}$ tag response using (6). Crosses indicate extracted values at the scatterer resonance frequency. Six displacements (from 0.5 to $3 \mathrm{~mm}$, with a step of $0.5 \mathrm{~mm}$ ) have been simulated. Displacements are towards the excitation.

If now a non-movable dielectric plate is added in front of the tag (which corresponds to the worst case as seen Fig. 3), with (3) and (6) the results plotted in Fig. 6 are obtained. The presence of an object prevents any measurement of the tag displacements in co-polarization has expected. Results obtained with (6) describe a stationary state, which corresponds to the presence of a non-movable plate. About the cross-polarization component, the condition $\left|C_{v h}\right| \gg\left|O_{v h}\right|$ is observed at each resonance frequency, which corresponds to the validity domain of (2) and (3). At this frequency, with (3), tag displacements can be extracted with a good accuracy. Contrary to the case without the plate, Fig. 6 shows that relation (3) which gives less accurate results outside the tag resonance frequency. In that configuration, with the dielectric plate, $\left|O_{v h}\right|$ is negligible (a backscattered E-field less than - 
$120 \mathrm{~dB}$ is obtained in simulation), but $\left|C_{v h}\right|$ is also small outside the frequencies of resonance [see Fig. 7(a)].

Another way to illustrate the effect of the dielectric plate on the displacement extraction is to use a time-gated technique to suppress the early time response of the signal. By doing this, it is possible to remove the structural mode of the crosspolarization tag's response. Fig. 7(a) shows a comparison of the spectral response of the entire signal and the gated signal corresponding to the antenna mode. It can be clearly seen that the power density of the signal is located around the tag resonance frequencies. Looking at Fig. 6(a), outside the tag resonance frequency, the displacements $d_{C}$ extracted from this gated signal are far from the expected values. This confirms the previous results and shows that the robustness of the detection is based on the scatterer resonances. It is also interesting to look at the co-polarization signal; in that case, by suppressing the early time response, the reflection of the signal on the dielectric plate can be removed (as well as the structural mode of the co-polarization tag's response). As this tag also operates in co-polarization (half of the power is backscattered in the co- polarization), Fig. 7(b) depicts the resonance on the time gating signal. By doing this, in relation to the total signal, the proportion of the signal backscattered from movable tag is increased. So it is then possible to extract displacement values that are in better agreement with the expected ones at the resonance frequency [see Fig. 6(b)]. . Unfortunately, co-polarization measurements cannot be implemented in practice, due to the presence of multiple objects surrounding the tag [28]. In such conditions, the tag's response cannot be separated from these multiple reflections.

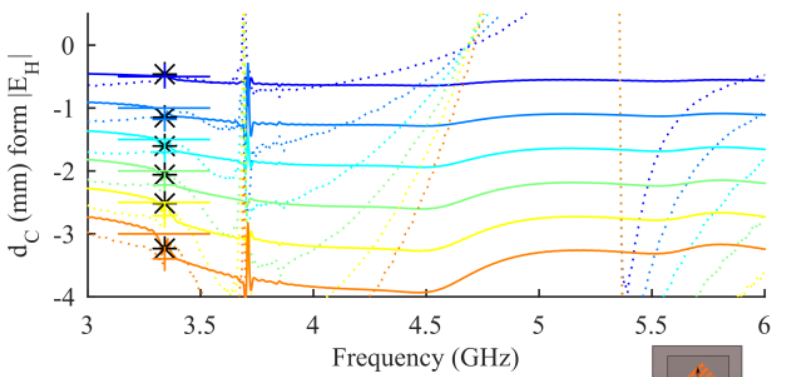

(a)

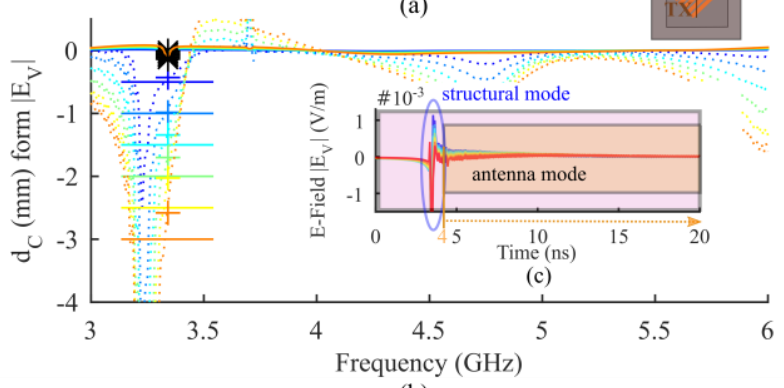

(b)

Fig. 6. Displacements of a depolarizing tag (single scatterer) based on shorted dipoles oriented at $45^{\circ}$ operating at $3.34 \mathrm{GHz}$ and simulated with a dielectric plate (see Fig. 3). (a) $d_{C}$ is derived from the cross-polarized E-field $E_{H}$ tag response (dash line) using (3). (b) $d_{C}$ is derived from the co-polarized E-field $E_{V}$ tag response (dash line) using (6). Asterisks indicate extracted values at the tag resonance frequency. Six displacements (from 0.5 to $3 \mathrm{~mm}$, with a step of $0.5 \mathrm{~mm}$ ) have been simulated. (c) Simulated time domain response for the scatterer in cross-polarization. The late time response of the signal $\left(\mathrm{t}>4 \mathrm{~ns}\right.$ ) has been used to extract $d_{C}$. The obtained curves (dotted line) are plotted in Figs. 6(a) and (b) for comparison.
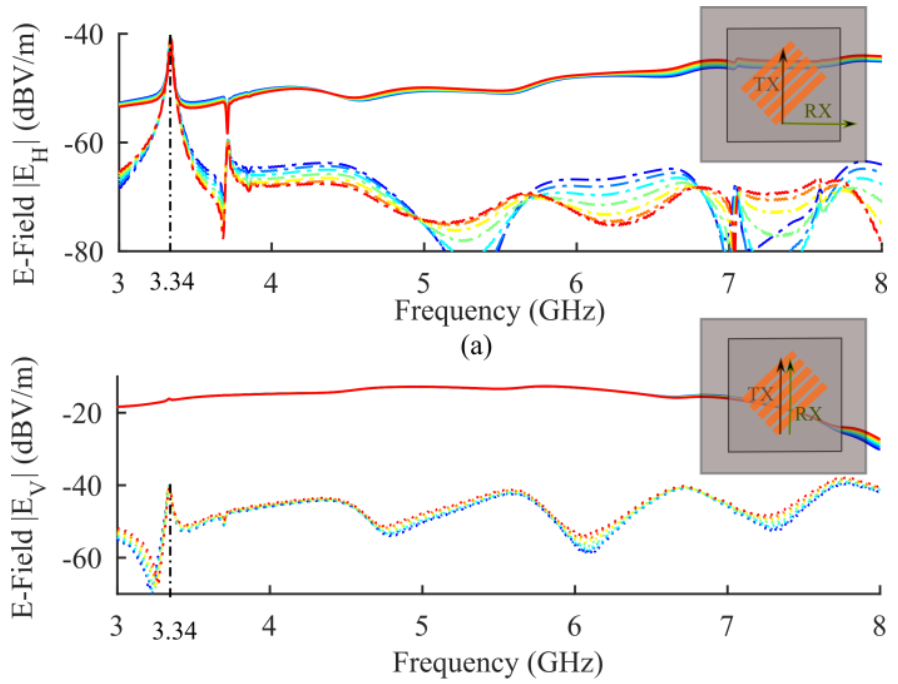

(b)

Fig. 7. Displacements of a depolarizing tag (single scatterer) based on shorted dipoles oriented at $45^{\circ}$ operating at $3.34 \mathrm{GHz}$ and simulated with a dielectric plate (see Fig. 3). Frequency-domain simulation of the E-Field response, (a) in cross-polarization, (b) in co-polarization. The dotted line corresponds to the late time response ( $\mathrm{t}>4 \mathrm{~ns}$ ) of the tag [see Fig. 6(c)].

Last configuration of two tags is now being presented. The same highly reflective plate of Roger is placed between the two tags and the feed (see Fig. 3). Tags are two scatterers, resonating at two different frequencies: $5.10 \mathrm{GHz}$ for the tag $\mathrm{n}^{\circ} 1$ and $3.34 \mathrm{GHz}$ for the tag $\mathrm{n}^{\circ} 2$. Simulation results are plotted in Fig. 8. Only the extraction of $d_{C}$ based on the crosspolarization component is shown. Again, the $d_{C}$ values extracted correspond to the imposed displacements when the resonance frequencies are considered.
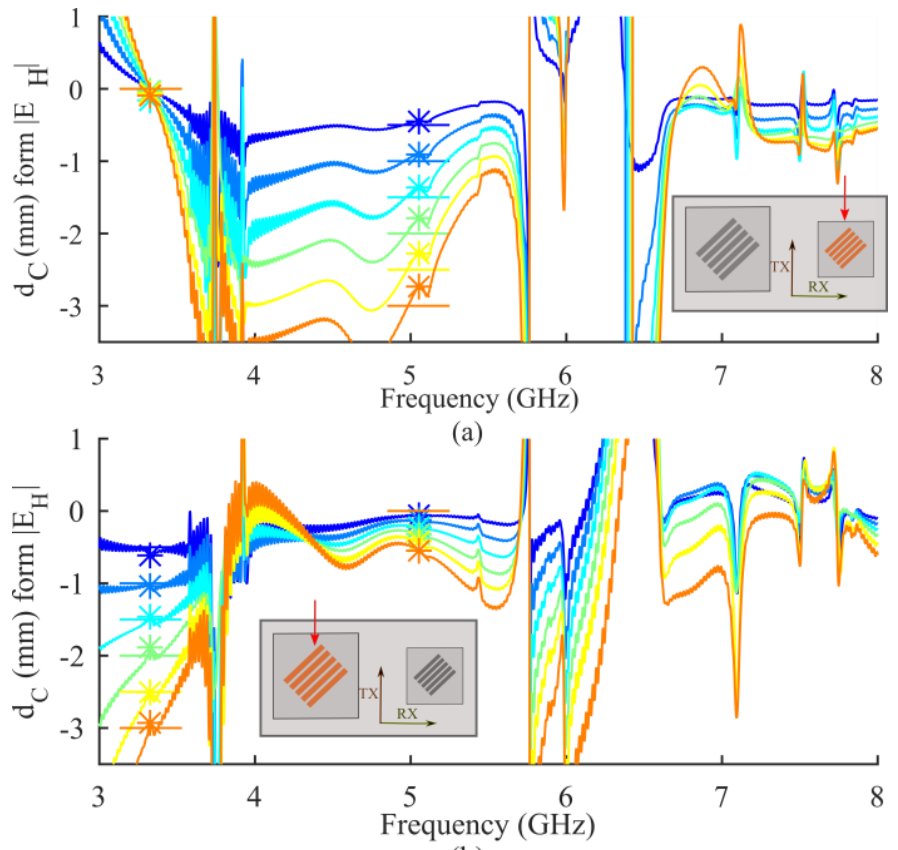

(b)

Fig. 8. Displacements of two depolarizing tags (single scatterer each) simulated with a dielectric plate (see Fig. 3 ). $d_{C}$ is derived from the crosspolarized E-field $E_{H}$ tag response using (3). (a) Tag n ${ }^{\circ} 1$ is moving (resonance frequency: $5.10 \mathrm{GHz}$ ), (b) tag $\mathrm{n}^{\circ} 2$ is moving (resonance frequency: $3.34 \mathrm{GHz}$ ). Asterisks indicate extracted values at the tag resonance frequencies. Six displacements (from 0.5 to $3 \mathrm{~mm}$, with a step of $0.5 \mathrm{~mm}$ ) have been simulated. The late time backscattered tag response ( $\mathrm{t}>4 \mathrm{~ns}$ ) has been used to extract $d_{C}$. 
This is observed in the two different cases: 1) tag $\mathrm{n}^{\circ} 1$ moves, the other objects are fixed and 2) tag $n^{\circ} 2$ moves, the other objects are fixed. Like previously, the presence of the dielectric plate prevents any measurement of the tag displacements with the co-polarized E-field signal (not seen). Simulations with movable objects and fixed or movable tag have also been carried out. Same observations have been done; by using the cross-polarization signal, it is possible to detect tag displacements, even if unknown and potentially movable objects are placed around the tags.

\section{EXPERIMENTAL VALIDATION}

In the theoretical part, some hypotheses have been done to work out a method for displacement detection based on the presence of scatterers placed on an object to monitor. These implications are meanly linked to the fact that common surrounding objects are no responding in cross-polarization. To validate the hypotheses introduced in Section III, a comparable study based on measurement results is presented. After having described the experimental protocol, measurements in an anechoic chamber are shown. The same measurements are performed in a real environment to evaluate the effect of the unknown surrounding objects. Then the accuracy of the approach with distance is discussed.

\section{A. Experimental protocol}

Unlike simulation, in measuring, the direct coupling $I_{v h}$ (or $\left.I_{v v}\right)$ is significant compared to the tag response. So, to characterize the direct coupling a first measurement of the environment without the tag (background or empty environment) is done. After that, with ${M^{\prime}}_{v h}^{\left(d_{C}\right)}(f)=S_{21}\left(f, d_{C}\right)$, and $M_{v v}^{\prime\left(d_{C}\right)}(f)=S_{11}\left(f, d_{C}\right)$, expressions (3) (crosspolarization configuration) and (6) (co-polarization configuration) can be derived for various physical displacements $d_{C}$ and $d_{O}$.

To validate the approach, an Agilent N5224A Vector Network Analyzer (VNA) has been used to act as a "reader". Fig. 9 shows the measurement bench used inside an anechoic chamber. The dual-access dual-polarization Satimo QH2000 antenna is connected to the VNA ports 1 and 2, in horizontal and vertical polarization, respectively. This antenna presents isolation greater than $30 \mathrm{~dB}$ between ports. The gain of the antennas is on the order of $12 \mathrm{dBi}$ between $1.5 \mathrm{GHz}$ and $8 \mathrm{GHz}$. The VNA output power is $-5 \mathrm{dBm}$.

The depolarizing tags introduced in [28] have been used in this study. The dimensions of these tags [the tag based on shorted dipoles oriented at $45^{\circ}$ (see Fig. 9), and the tag based on dual-L resonators (see Fig. 1)] are given in [28, Tables I and II].

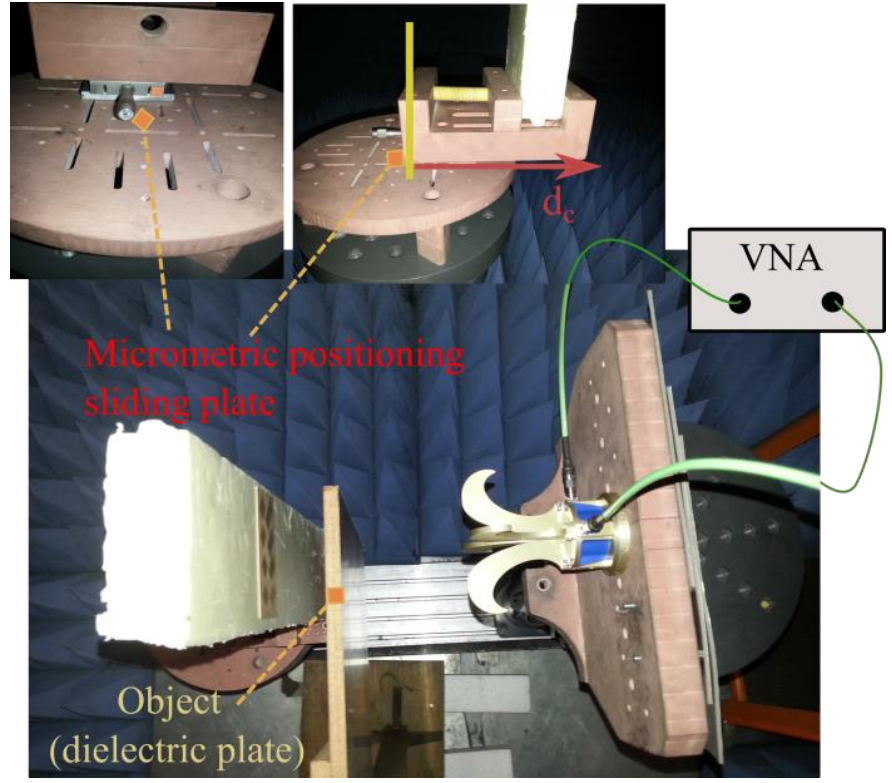

Fig. 9. Measurement bench used in an anechoic chamber for displacement measurements. Photograph of depolarizing tag based on shorted dipole. A dielectric plate of CARP $\left(\varepsilon_{r}=5.7\right)$ of $10 \mathrm{~mm}$ thickness is placed between the tag and the antenna.

\section{B. Measurements in an anechoic chamber}

First, the tag (8 scatterers) based on shorted dipoles oriented at $45^{\circ}$ is placed on a support (foam) attached to a micrometric positioning sliding plate, at a distance $D$ equal to $20 \mathrm{~cm}$ of the horn antenna (see Fig. 9). To determine the minimum displacement distance that can be extracted with this approach, displacements from $0.1 \mathrm{~mm}$ to $0.5 \mathrm{~mm}$ with a step of $0.1 \mathrm{~mm}$ have been measured in anechoic chamber (not seen here). A minimum displacement of $0.5 \mathrm{~mm}$ has been assessed from these preliminary measurement results. So the smallest displacement considered are $0.5 \mathrm{~mm}$, and the total translation range is $3 \mathrm{~mm}$. Fig. 10 shows the displacement results extracted from the measurements. The results show very good agreement between the expected values and the processed results.

As expected in simulation and in theory, (3) gives accurate results of the measured displacements, especially around the tag resonance frequencies [see Fig. 10(b)]. It is also interesting to see in Figs. 10(a) and (c) that near resonance frequencies (on a range higher than $150 \mathrm{MHz}$ ), the curve corresponding to the extracted value $d_{C}$ is smooth and flat, that is to say less affected to the noise. If this result is compared with the equivalent simulation case plotted Fig. 5, measurement it is difficult to extract the displacement value outside tag resonance frequencies. This example shows that in practice even in a configuration closed to the simulation (tag alone), measurement errors and the presence of supports (even with very low contribution like the one used) have a non-negligible impact on the results. 

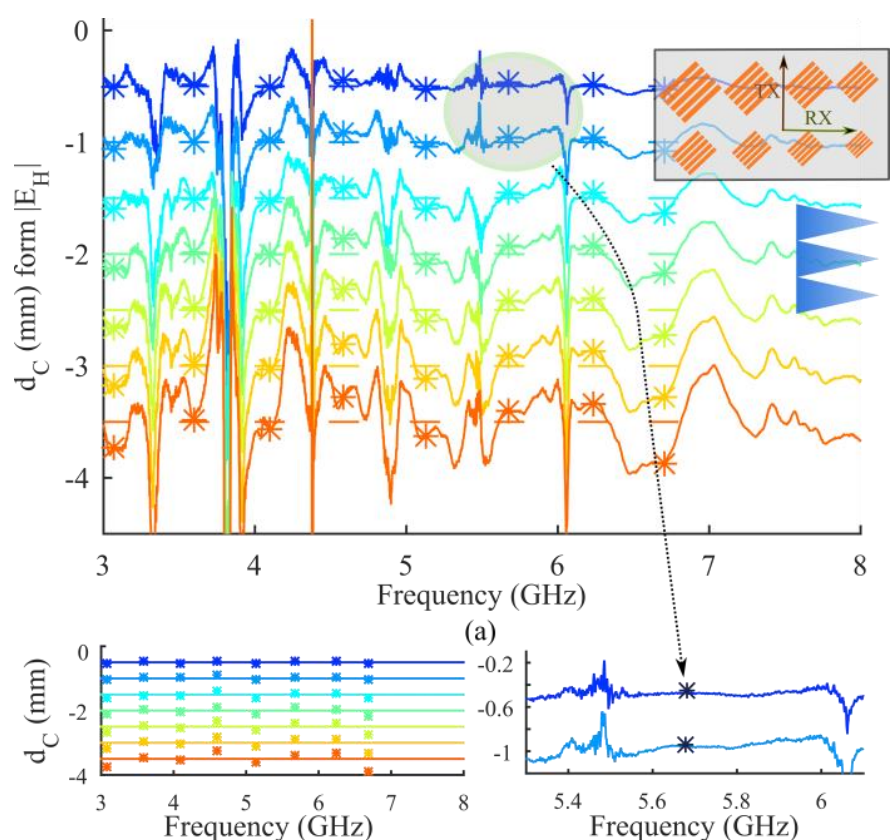

(b)

(a)

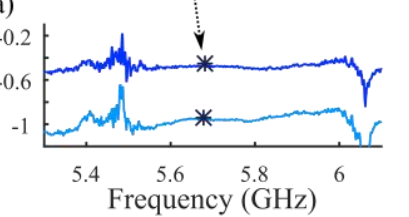

(c)

Fig. 10. Displacements of a depolarizing tag (8 scatterers) based on shorted dipoles oriented at $45^{\circ}$ operating between 3.34 to $6.8 \mathrm{GHz}$. (a) $d_{C}$ is derived from the measurement of $S_{21}$ parameter in anechoic chamber (see Fig. 9) and using (3). (b) Same than (a) but with only the expected values and the extracted values (asterisks) at the tag resonance frequencies. Seven displacements (from $0.5 \mathrm{~mm}$ to $3.5 \mathrm{~mm}$, with a step of $0.5 \mathrm{~mm}$ ) have been measured. (c) Zoom around a resonance frequency.

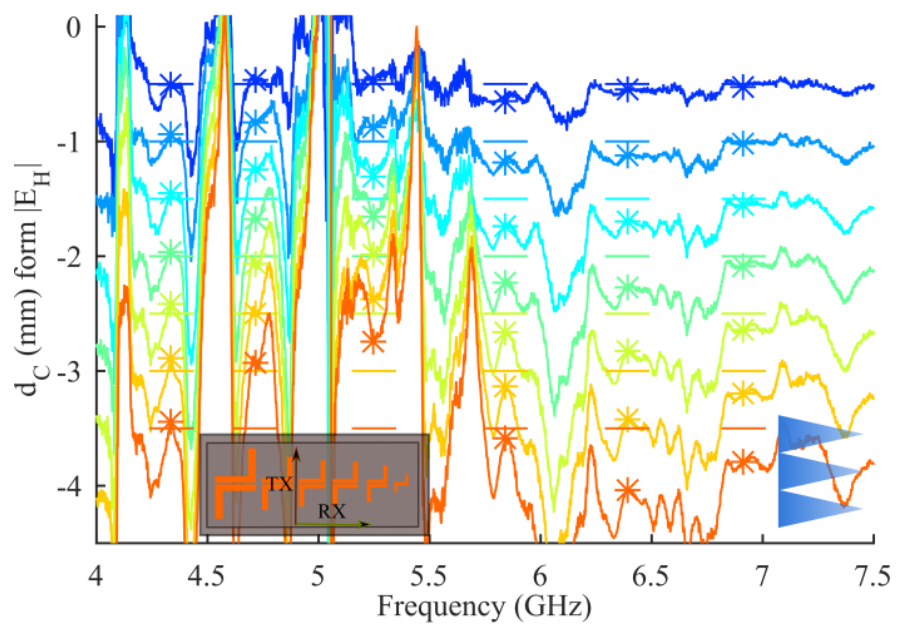

(a) (b)

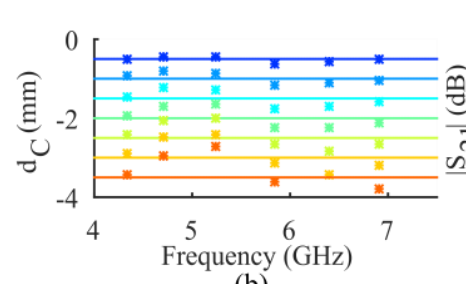

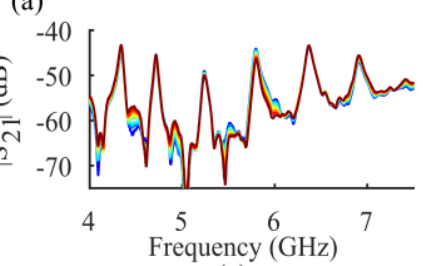

(c)
Fig. 11. Displacements of a depolarizing tag (6 scatterers) based on dual-L resonators operating between 4.3 to $6.9 \mathrm{GHz}$ measured with a dielectric plate of CARP (see Fig. 9). (a) $d_{C}$ is derived from the measurement of $S_{21}$ parameter in anechoic chamber and using (3). (b) Same than (a) but with only the expected values and the extracted values (asterisks) at the tag resonance frequencies. Seven displacements (from 0.5 to $3.5 \mathrm{~mm}$, with a step of $0.5 \mathrm{~mm}$ ) have been measured. (c) Cross-polarization measurements for de-polarizing tag for the seven distances. The results are obtained by subtracting the measurement of the tag with the measurement of the empty environment.
To see the limits of the approach, several objects have been added around the tag, and different configurations have been tried. First, a dielectric plate of phenolic resin laminate of 10 $\mathrm{mm}$ thickness (CARP) is placed between the tag and the reader antenna (see Fig. 9). With a high permittivity of 5.7, the configuration with CARP is the worst case that this paper has tried; the impact on the measurement is more significant compared to other materials such as PTFE, PET, or Kapton. Measurement results are plotted in Fig. 11. Again, (3) gives good results of the tag displacements when the resonance frequencies of the tag are considered [see Fig. 11(b)]. Fig. 11(c) shows the peak apex used to demine accurately the resonance frequencies to derive $d_{c}$.

These results show that the approximation $\left|C_{v h}\right| \gg\left|O_{v h}\right|$ is satisfied at the tag resonance frequencies. Comparing these results [Fig. 11(b)] with the one obtained without any high dielectric plate [Fig. 10(b)], we see that the presence of the plate degrades the performance, notably outside the resonance frequencies, like it was observed in simulation.

A configuration with two scatterers (one is moving) has also been tested (see Fig. 12). The results are plotted in Fig. 13. As expected, the displacements can be obtained by looking at the frequency of resonance $(4.16 \mathrm{GHz})$ of the movable tag (tag $\mathrm{n}^{\circ} 1$ in Fig. 12). However, when we look at the frequency of the second resonator (tag $\mathrm{n}^{\circ} 2$ which is fixed during the measurement), a small variation of around $0.5 \mathrm{~mm}$ is seen. This means that the contribution of the signal from the movable tag at the frequency of the second resonator has small impact on the total signal. Again, these results are in very good agreement with the ones obtained in simulation and plotted in Fig. 8(b).

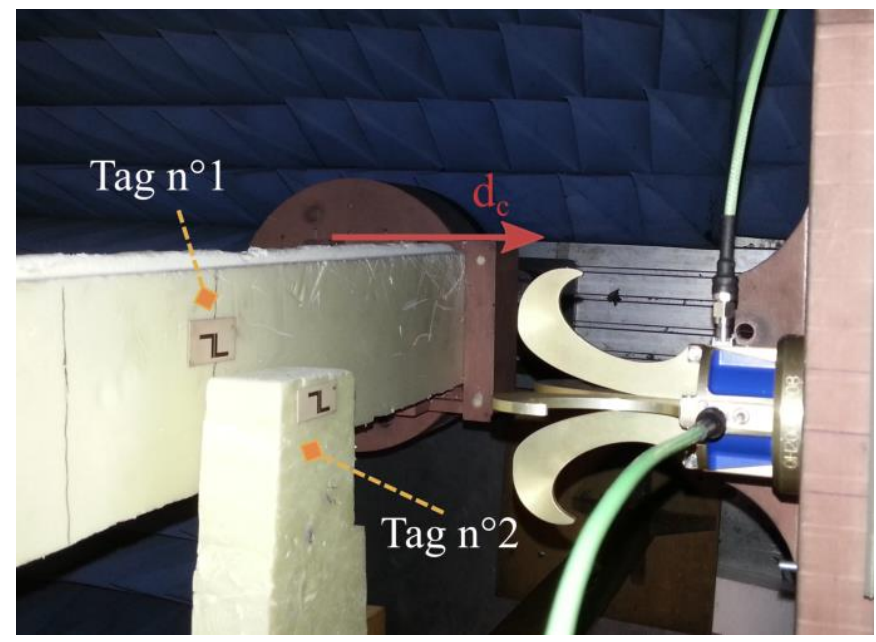

Fig. 12. Measurement bench used in an anechoic chamber for displacement measurements. Configuration with two tags.

To validate the improvement of cross-polarization measurements, co-polarization tags have been measured in the same conditions. As expected, the extraction of the displacement values cannot be done with enough accuracy. Results (not shown here) comparable with the ones plotted in Fig. 6(b) have been observed when a dielectric plate is placed between the tag and the antenna. Without the plate, information on the tag displacement cannot be obtained with enough accuracy even if time-gating is applied. 


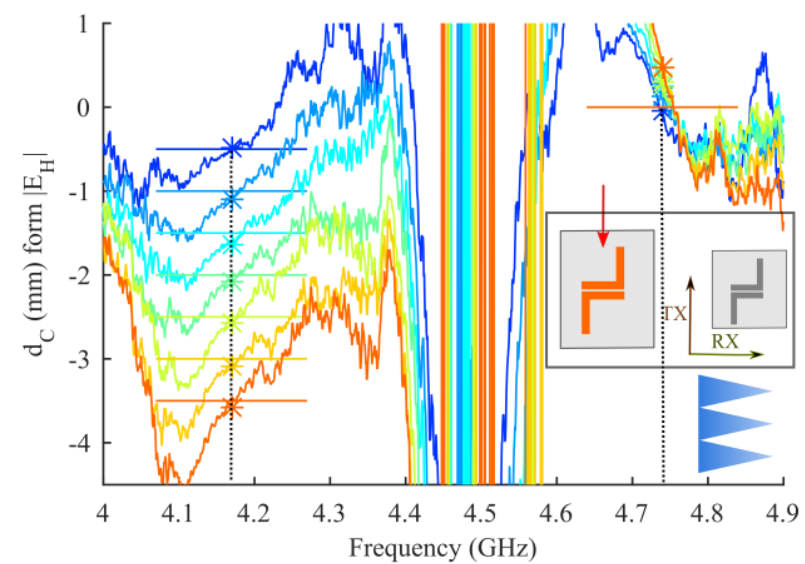

Fig. 13. Displacements of two depolarizing tag (single scatterer each) based on dual-L resonators operating at 4.16 and $4.72 \mathrm{GHz}$ respectively. $d_{C}$ is derived from the measurement of $S_{21}$ parameter in anechoic chamber (see Fig. 11) using (3). Only the tag with a resonance frequency of $4.16 \mathrm{GHz}$ is moving. Seven displacements (from $0.5 \mathrm{~mm}$ to $3.5 \mathrm{~mm}$, with a step of $0.5 \mathrm{~mm}$ ) have been measured.

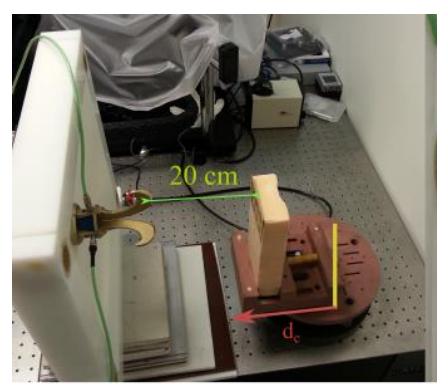

(a)

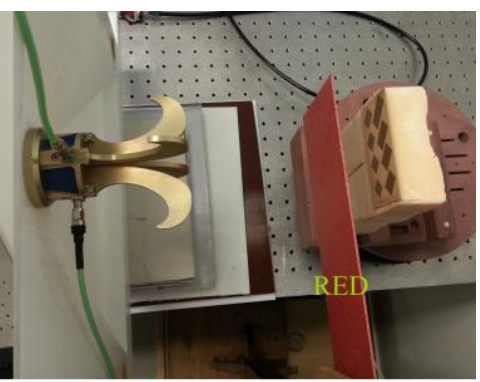

(b)
Fig. 14. (a) Measurement bench used in a room (real environment) for displacement measurements. (b) A dielectric plate (RED test sample, $\varepsilon_{r}=4.25$, $\tan \delta=0.0182)$ of $1.96 \mathrm{~mm}$ thickness is placed between the tag and the antenna.
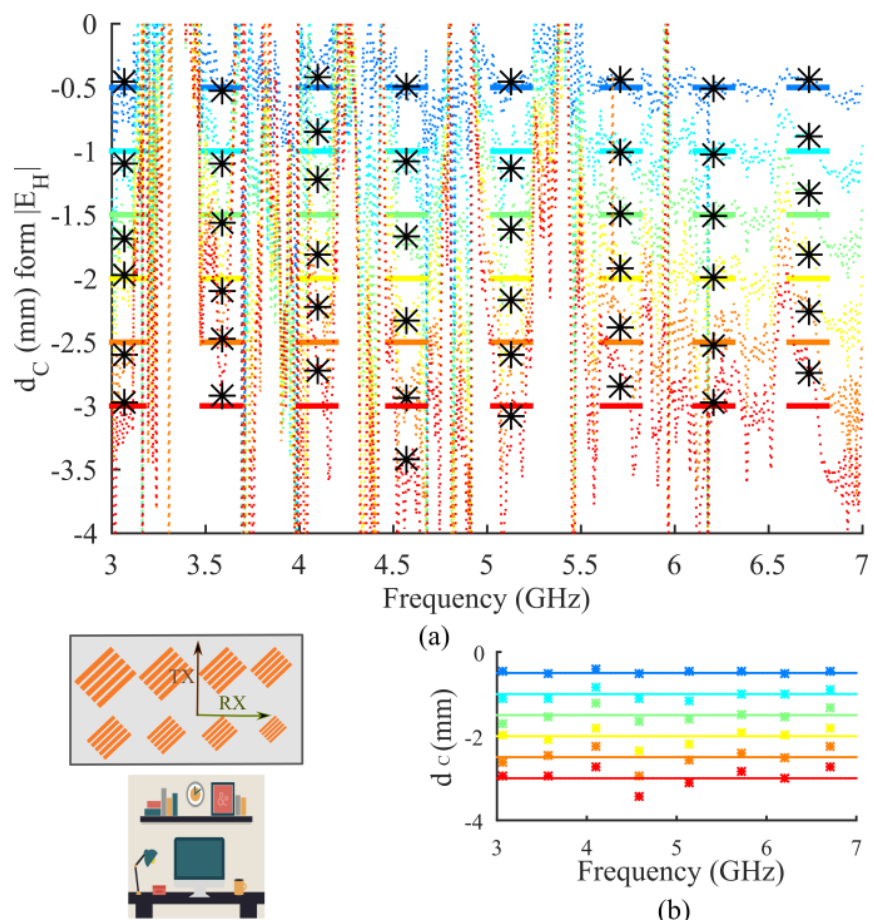

(a)

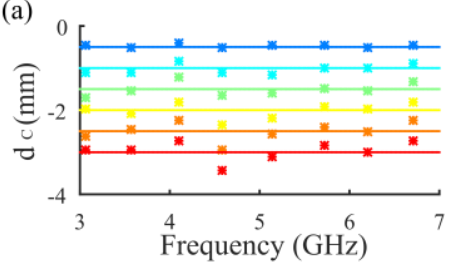

(b)

Fig. 15. Displacements of a depolarizing tag (8 scatterers) based on shorted dipoles oriented at $45^{\circ}$ operating between 3.34 to $6.8 \mathrm{GHz}$. (a) $d_{C}$ is derived from the measurement of $S_{21}$ parameter outside anechoic chamber [see Fig. 14(a)] and using (3). (b) Same than (a) but with only the expected values and the extracted values (asterisks) at the tag resonance frequencies.

\section{Measurements in a real environment}

Then, chipless tags have been measured in a room containing tables, chairs, and many metal elements. Photographs of the measurement environment for the tag alone and the tag with a dielectric plate (RED test sample) are presented, respectively, in Figs. 14(a) and (b). Corresponding measurement results are given in Figs. 15 and 16. The displacement values can be extracted at the tag's resonance frequencies with good accuracy. However, it is clear that outside these frequencies is a much distorted signal compared to the anechoic chamber set up. The RED sample has been placed behind the tag and between the tag and the antenna [worst configuration, see Fig. 14(b)]. Figs. 16(b) and (d) show the obtained results. In both configuration measurements of small displacements can be obtained with good accuracy. However, when the dielectric plate is in front of the tag, larger displacements are more difficult to measure with accuracy. This experimentation shows the limits of the approach with this type of tag. To improve the performance, scatters with higher quality factor, and more elaborate post-treatment technique such the one based on short-time Fourier transform [29] could be used.

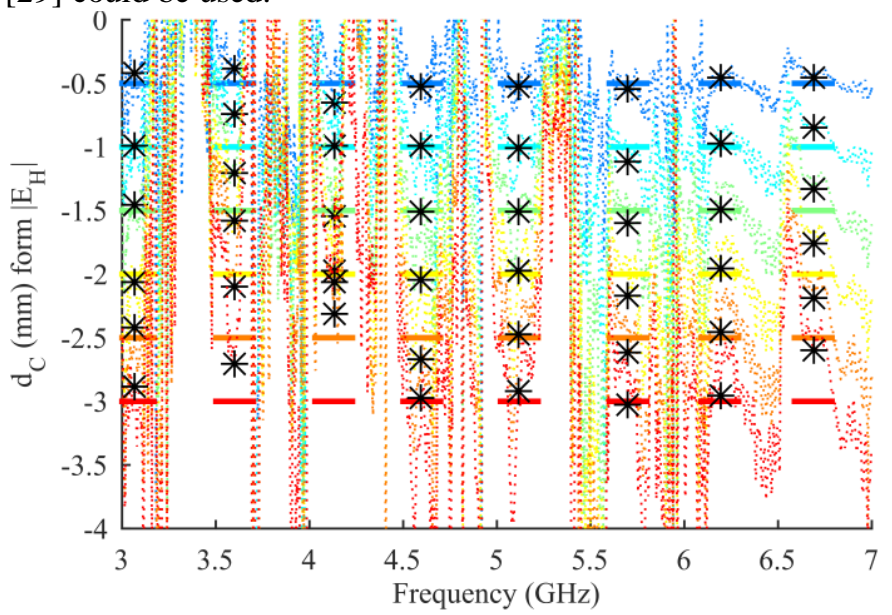

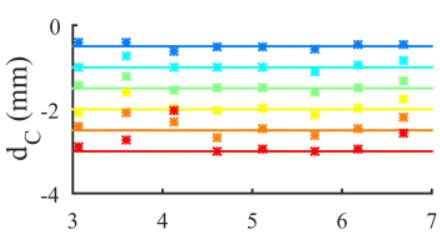

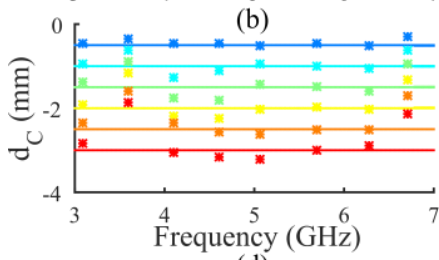

(d) (a)

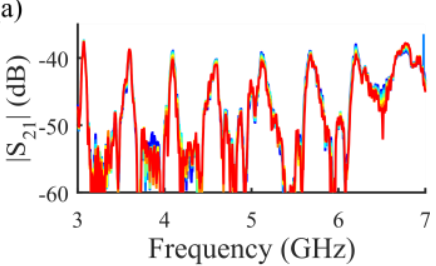

(c)
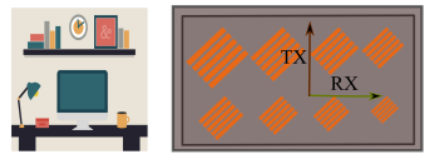

Fig. 16. Displacements of a depolarizing tag (8 scatterers) based on shorted dipoles oriented at $45^{\circ}$ operating between 3.34 to $6.8 \mathrm{GHz}$ measured outside anechoic chamber with a dielectric plate of RED [see Fig. 14(b)]. (a) $d_{C}$ is derived from the measurement of $S_{21}$ parameter using (3) - the RED sample is placed between the tag and the antenna. (b) Same than (a) but with only the expected values and the extracted values (asterisks) at the tag resonance frequencies. (c) Cross-polarization measurements for de-polarizing tag for the six distances. (d) Same than (b) but the RED sample is behind the tag.

\section{Accuracy of the approach, effect of the distance}

Table I gives the relative error obtained in simulation, but also inside and outside anechoic chamber. The error was 
computed from the simulation and measurement results presented in Figs. 5, 6, 8, 10, 12, 13, 15-16. The reference values considered are the imposed displacements (the horizontal bares shown in the figures); and the comparison is done with the extracted results obtained with the analytical formulas (asterisks plotted in the figures). The tag is placed at a distance of $20 \mathrm{~cm}$ from the antenna. These results show that typical displacements of $0.5 \mathrm{~mm}$ can be monitored. Whatever the configuration used, relative errors are of the order of a few percent or less.

Table 1 shows also the error value obtained by deriving the median of all the extracted distances for tags based on multiresonators (with 6 and 8 resonators). The overall error decreases significantly when all these extracted values (median over 6 or 8 values) are used to compute the displacement. Indeed, a relative error of less than $\pm 9 \%$ for all the real-environment configurations is obtained regardless of the displacements characterized (between 0.5 and $3 \mathrm{~mm}$ - with errors less than $\pm 3 \%$ for $3 \mathrm{~mm}$ displacement). Note that a relative error of $9 \%$ for a displacement of $0.5 \mathrm{~mm}$ corresponds to an error of $\pm 45 \mu \mathrm{m}$. In anechoic chamber, an error of only few $\mu \mathrm{m}$ is obtained even in the presence of a dielectric plate.

TABLE I

DISPLACEMENT EXTRACTIONS - RELATIVE ERROR COMPARISON (\%)

\begin{tabular}{|c|c|c|c|c|c|c|c|c|}
\hline \multicolumn{3}{|c|}{$\begin{array}{l}d_{c}(\mathrm{~mm}) \\
\text { Configuration }\end{array}$} & 0.5 & 1 & 1.5 & 2 & 2.5 & 3 \\
\hline \multirow{4}{*}{ 总 } & \multicolumn{2}{|c|}{ Fig. 5 - Air } & $<0.01$ & $<0.01$ & $<0.01$ & $<0.01$ & $<0.01$ & $<0.01$ \\
\hline & \multicolumn{2}{|c|}{ Fig. 6 - Roger } & -8.6 & 13.1 & 6.3 & 2.9 & 1.1 & 7.4 \\
\hline & \multirow{2}{*}{$\begin{array}{l}\text { Fig. } \\
8(a)- \\
2 \text { tags }\end{array}$} & $f_{1}$ & 24.8 & 4.7 & -2.3 & -5.9 & 0.4 & -2.3 \\
\hline & & $f_{2} *$ & $<0.1$ & 0.1 & 0.2 & 0.3 & 0.4 & 0.5 \\
\hline \multirow{6}{*}{ 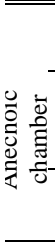 } & \multirow{2}{*}{$\begin{array}{l}\text { Fig. } 10 \\
\text { - Air }\end{array}$} & $\operatorname{Res} n^{\circ} 1$ & 2.5 & 4.4 & 6.3 & 6.2 & 6.2 & 5.6 \\
\hline & & Median & -0.8 & -1.2 & 1.7 & 0.1 & 0.2 & 0.2 \\
\hline & \multirow{2}{*}{$\begin{array}{l}\text { Fig. } 12 \\
- \\
\text { CARP }\end{array}$} & $\operatorname{Res} n^{\circ} 1$ & -0.4 & -6.3 & -4.1 & -3.2 & $\begin{array}{l}-3.7 \\
-3.7\end{array}$ & -1.9 \\
\hline & & Median & 0.6 & -1.8 & -0.1 & 0.6 & 1.0 & 0.9 \\
\hline & \multirow{2}{*}{$\begin{array}{l}\text { Fig. } 13 \\
-2 \text { tags }\end{array}$} & $f_{1}$ & -1.7 & 10.0 & 8.8 & 3.5 & 2.6 & 3.2 \\
\hline & & $f_{2} *$ & $<0.1$ & 0.1 & 0.2 & 0.2 & 0.3 & 0.4 \\
\hline \multirow{6}{*}{ 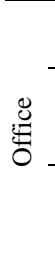 } & \multirow{2}{*}{$\begin{array}{l}\text { Fig. } 15 \\
\text { - Air }\end{array}$} & $\operatorname{Res} n^{\circ} 1$ & -8.5 & 10.6 & 12.2 & -0.9 & 4.0 & -1.0 \\
\hline & & Median & -8.9 & 9.0 & 5.6 & 2.2 & 1.4 & 1.7 \\
\hline & \multirow{2}{*}{$\begin{array}{l}\text { Fig. } \\
\text { 16(b) - } \\
\text { Red }\end{array}$} & Res n ${ }^{\circ} 1$ & -5.6 & -3.5 & -6.3 & -4.5 & -6.2 & -5.9 \\
\hline & & Median & 8.4 & -3.0 & -2.1 & -0.4 & -2.5 & 1.4 \\
\hline & \multirow{2}{*}{$\begin{array}{l}\text { Fig. } \\
\text { 16(d) - } \\
\text { Red }\end{array}$} & $\operatorname{Res} n^{\circ} 1$ & -15.7 & -1.2 & -2.9 & 3.5 & -3.0 & -3.6 \\
\hline & & Median & 6.1 & -0.4 & 0.5 & 0.3 & -1.9 & -3.0 \\
\hline
\end{tabular}

*Absolute error (mm)

The maximum reading distance and the impact of the distance $D$ on the accuracy of the displacements extraction have been investigated. The tag has been placed at different distances (from $20 \mathrm{~cm}$ to $100 \mathrm{~cm}$, with a step of $20 \mathrm{~cm}$ ) from the antenna. The depolarizing tag ( 8 scatterers) based on shorted dipoles oriented at $45^{\circ}$ has been measured in anechoic chamber. Again, the extraction of the displacements has been done for each of the 8 frequencies of resonance ( 8 extracted values for each displacement). The median value has been computed. Then the relative error and the standard deviation have been derived. Table II shows the obtained results. We observed that the standard deviation increased significantly with the distance. The relative error increases slowly and the obtained error remains acceptable (less than $\pm 15 \%$ ) in that range. However for accurate measurements, a maximum read range of $40 \mathrm{~cm}$ has to be chosen. For this distance a resolution of $30 \mu \mathrm{m}$ is obtained in anechoic chamber.
TABLE II

DISPLACEMENT EXTRACTION ACCURACY WITH DISTANCE

\begin{tabular}{cccccc}
\hline $\mathbf{d}(\mathbf{c m})$ & $\mathbf{0 . 5}$ & $\mathbf{1}$ & $\mathbf{1 . 5}$ & $\mathbf{2}$ & $\mathbf{2 . 5}$ \\
\hline \multicolumn{5}{c}{ Relative error $(\%)$} \\
\hline 20 & -6.88 & 0.65 & -1.29 & 1.10 \\
\hline 40 & -3.92 & -3.24 & -2.70 & -2.09 & 1.88 \\
\hline 60 & -2.61 & -14.03 & -4.50 & -4.57 & -0.90 \\
\hline 80 & 2.790 & 14.70 & -0.08 & -10.71 & \\
\hline 100 & -1.76 & -15.48 & -4.81 & -6.41 & \\
\hline \hline & & Standard deviation & $(\mathrm{mm})$ & \\
\hline 40 & 0.029 & 0.056 & 0.084 & 0.110 & \\
\hline 60 & 0.047 & 0.054 & 0.049 & 0.090 & 0.074 \\
\hline 80 & 0.186 & 0.265 & 0.284 & 0.524 & 0.390 \\
\hline 100 & 0.342 & 0.383 & 0.525 & 0.591 & \\
\hline & 1.293 & 0.449 & 0.618 & 0.916 & \\
\hline
\end{tabular}

\section{V.CONCLUSION}

This work has presented a displacement detection technique compatible with the use of common frequency-coded chipless RFID tags. The true performance of the introduced technique in term of precision and accuracy in real environment has been assessed. The possibility to conceive chipless RFID system as measurement instrument has been shown. The measurement of the distance variation of chipless RFID tags surrounded by unknown objects gives satisfactory results and shows the reliability of the approach. The use of chipless RFID scatterers operating in cross-polarization proved to be an effective solution for a totally passive, wireless, and non-line-of-sight sensing of displacements with a resolution of less than one millimeter. Moreover, this sensing functionality can be implemented on pre-designed chipless RFID tag operating in cross-polarization without decreasing their coding capacity. In addition to the tag ID, by post processing, it is possible to get the information on the tag displacement.

It has been shown that the displacement measurements of unknown objects in unknown environments are possible and are obtained directly without the use of complex calibration process or even calibration curves, which are difficult to implement in practice.

\section{REFERENCES}

[1] A. Costanzo, D. Masotti, T. Ussmueller, and R. Weigel, "Tag, You're It: Ranging and Finding via RFID Technology," IEEE Microw. Mag., vol. 14, pp. 36-46, 2013.

[2] E. Perret, "Radio Frequency Identification and Sensors: From RFID to Chipless RFID," Wiley-ISTE, 2014.

[3] G. Marrocco, "RFID grids: Part I-Electromagnetic theory," IEEE Trans. Antennas Propag., vol. 59, pp. 1019-1026, 2011.

[4] S. Caizzone and G. Marrocco, "RFID Grids: Part IIExperimentations," IEEE Trans. Antennas Propag., vol. 59, pp. 28962904, 2011.

[5] B. S. Cook, R. Vyas, S. Kim, T. Thai, T. Le, A. Traille, H. Aubert, and M. M. Tentzeris, "RFID-Based Sensors for Zero-Power Autonomous Wireless Sensor Networks," IEEE Sensors J., vol. 14, pp. 2419-2431, 2014.

[6] R. Bhattacharyya, C. Floerkemeier, and S. Sarma, "Towards tag antenna based sensing - An RFID displacement sensor," in IEEE Int. Conf. RFID, 2009, pp. 95-102.

[7] S. Caizzone and G. Marrocco, "RFID-grids for deformation sensing " in IEEE Int. Conf. RFID, Orlando (USA), 2012, pp. 130 - 134.

[8] C. Occhiuzzi and G. Marrocco, "Precision and Accuracy in UHF-RFID Power Measurements for Passive Sensing," IEEE Sensors J., vol. 16, pp. 3091-3098, 2016.

[9] A. Guillet, A. Vena, E. Perret, and S. Tedjini, "Design of a chipless RFID sensor for water level detection " in 15th Int. Symp. Antenna Technol. and Appl. Electromagnetics, Toulouse, France, 2012, pp. 1-4. 
[10] T. Bauernfeind, K. Preis, G. Koczka, S. Maier, and O. Biro, "Influence of the Non-Linear UHF-RFID IC Impedance on the Backscatter Abilities of a T-Match Tag Antenna Design," IEEE Trans. Magn., vol. 48, pp. 755758, 2012.

[11] E. Perret, S. Tedjini, and R. Nair, "Design of Antennas for UHF RFID Tags," Proc. IEEE, vol. 100, pp. 2330 - 2340, 2012.

[12] A. Pouzin, T. P. Vuong, S. Tedjini, M. Pouyet, J. Perdereau, and L. Dreux, "Determination of measurement uncertainties applied to the RCS and the differential RCS of UHF passive RFID tags," in IEEE APSURSI, 2009, pp. $1-4$.

[13] D. G. Kuester, D. R. Novotny, J. R. Guerrieri, A. Ibrahim, and Z. B. Popovic, "Simple Test and Modeling of RFID Tag Backscatter," IEEE Trans. Microw. Theory Techn., vol. 60, pp. 2248-2258, 2012.

[14] S. Peradovic and N. C. karmakar, "Chipless RFID tag with Integrated Sensor," in IEEE Int. Conf. in Sensors, 2010, pp. 1277-1281.

[15] S. Shrestha, M. Balachandran, M. Agarwal, V. V. Phoha, and K. Varahramyan, "A Chipless RFID Sensor System for Cyber Centric Monitoring Applications," IEEE Trans. Microw. Theory Techn., vol. 57, pp. 1303 - 1309, may 2009.

[16] A. Vena, E. Perret, S. Tedjini, D. Kaddour, A. Potie, and T. Baron, "A Compact Chipless RFID Tag with Environment Sensing Capability," in IEEE IMS, Montréal (Canada), 2012, pp. 1-3.

[17] R. S. Nair, E. Perret, S. Tedjini, and T. Baron, "A Group Delay Based Chipless RFID Humidity Tag Sensor Using Silicon Nanowires," IEEE Microw. Compon. Lett., vol. 12, pp. 729-732, 2013.

[18] H. Aubert, F. Chebila, M. Jatlaoui, T. Thai, H. Hallil, A. Traille, S. Bouaziz, A. Rifa, P. Pons, P. Menini, and M. Tentzeris, "Wireless sensing and identification of passive electromagnetic sensors based on millimetrewave FMCW RADAR," in IEEE Int. Conf. RFID-TA, 2012, pp. 398-403.

[19] E. Perret, R. S. Nair, E. Bel Kamel, A. Vena, and S. Tedjini, "Chipless RFID tags for passive wireless sensor grids," in XXXIth URSI GASS, 2014, pp. 1-4.

[20] L. Xinqun, W. Clegg, D. F. L. Jenkins, and B. Liu, "Polarization interferometer for measuring small displacement," IEEE Trans. Instrum. Meas., vol. 50, pp. 868-871, 2001.

[21] B. G. Zagar, "A laser-interferometer measuring displacement with nanometer resolution," IEEE Trans. Instrum. Meas., vol. 43, pp. 332-336, 1994.

[22] P. Joongsuk and N. Cam, "Development of a new millimeter-wave integrated-circuit sensor for surface and subsurface sensing," IEEE Sensors J., vol. 6, pp. 650-655, 2006.

[23] K. Seoktae and N. Cam, "A displacement measurement technique using millimeter-wave interferometry," IEEE Trans. Microw. Theory Techn., vol. 51, pp. 1724-1728, 2003.

[24] W. Massagram, N. Hafner, V. Lubecke, and O. Boric-Lubecke, "Tidal Volume Measurement Through Non-Contact Doppler Radar With DC Reconstruction," IEEE Sensors J., vol. 13, pp. 3397-3404, 2013.

[25] A. Lazaro, D. Girbau, and R. Villarino, "Analysis of vital signs monitoring using an IR-UWB radar," Progress Electromagn. Res., pp. 265-284, 2010.

[26] A. Vena, E. Perret, S. Tedjini, G. E. P. Tourtollet, A. Delattre, F. Garet, and Y. Boutant, "Design of Chipless RFID Tags Printed on Paper by Flexography," IEEE Trans. Antennas Propag., vol. 61, pp. 5868-5877, 2013.

[27] M. Garbati, R. Siragusa, and E. Perret, "Low Cost Low Sampling Noise UWB Chipless RFID Reader," in IEEE IMS 2015, Phoenix (USA), 2015, pp. 1-4.

[28] A. Vena, E. Perret, and S. Tedjini, "A Depolarizing Chipless RFID Tag for Robust Detection and Its FCC Compliant UWB Reading System," IEEE Trans. Microw. Theory Techn., vol. 61, pp. 2982 - 2994, 2013.

[29] A. Ramos, E. Perret, O. Rance, S. Tedjini, A. Lazaro, and D. Girbau, "Temporal Separation Detection for Chipless Depolarizing FrequencyCoded RFID," IEEE Trans. Microw. Theory Techn., vol. 64, pp. 23262337, 2016..

[30] R. Bhattacharyya, C. Floerkemeier, S. Sarma, and D. Deavours, "RFID tag antenna based temperature sensing in the frequency domain," in IEEE Int. Conf. RFID, 2011, pp. 70-77.

[31] S. Manzari, C. Occhiuzzi, S. Nawale, A. Catini, C. Di Natale, and G. Marrocco, "Polymer-doped UHF RFID tag for wireless-sensing of humidity," in IEEE Int. Conf. RFID, 2012, pp. 124-129.

[32] J. Siden, X. Zeng, T. Unander, A. Koptyug, and H. E. Nilsson, "Remote Moisture Sensing utilizing Ordinary RFID Tags," in IEEE Sensors, Atlanta (USA), 2007, pp. 308-311.

[33] R. Bhattacharyya, C. Floerkemeier, and S. Sarma, "Towards tag antenna based sensing - An RFID displacement sensor," in IEEE Int. Conf. RFID, 2009, pp. 95-102.
[34] R. Bhattacharyya, C. Floerkemeier, and S. Sarma, "RFID tag antenna based sensing: does your beverage glass need a refill?," in IEEE Int. Conf. RFID, 2010, pp. 126-133. 